\title{
New Strategies in Clinical Care of Skin Wound Healing
}

\author{
C.I. Günter H.-G. Machens \\ Clinic for Plastic and Hand Surgery, University Hospital rechts der Isar, Technische Universität München, \\ Munich, Germany
}

\section{Key Words}

Erythropoietin $\cdot$ Growth factors $\cdot$ Tissue engineering $\cdot$ Gene therapy $\cdot$ Stem cells

\begin{abstract}
The prevalence of chronic wounds is closely correlated to the aging population and so-called civilizational diseases. Therefore, they are causing morbidity and mortality of millions of patients worldwide, with an unbroken upward trend. As a consequence, chronic wounds induce enormous and rapidly growing costs for our health care systems and society in general. Thus, medically effective and cost-efficient treatment methods are urgently needed. Methods of 'regenerative medicine' might offer innovative scientific solutions, including the use of stem cells, growth factors and new bioactive materials. These tools are experimentally well described but clinically poorly performed. The main reasons for this are both legislative and economic. This review describes stateof-the-art techniques, up-to-date research projects, innovative preclinical and clinical approaches in wound care, and activities to translate these innovative techniques into clinical routine.

Copyright $\odot 2012$ S. Karger AG, Basel
\end{abstract}

\section{Introduction}

The skin is the largest organ of the human body. For a long time, however, it was merely seen as a simple coat just covering the body. Nowadays we know more about the multitude of physiological organic functions it has to fulfill. These tasks include mechanistic, metabolic, regenerative, energetic and immunological aspects.

Skin tissue was the first tissue to have been successfully tissue-engineered in vitro and to have also been successfully translated back into clinical application. Not only due to its easy accessibility but also because of the fact that the skin is one of the most active and continuously regenerating organs, it is a prime target for regenerative therapies and, in addition, a fascinating model from which to learn more about the human body's intrinsic regenerative mechanisms.

Modern wound care starts to integrate and support the body's own regenerative capacities more systematically. Methods of 'regenerative medicine' are in the scientific focus, including the use of stem cells, growth factors and new bioactive materials, and combinations of these methods. Stem cells of different origins are currently under active observation, mostly in preclinical research projects and increasingly in early-phase clinical trials. Due to the (European) legislative situation for 'advanced

Christina Irene Günter, MD

Clinic for Plastic and Hand Surgery

University Hospital rechts der Isar, Technische Universität München

Ismaninger Strasse 22, DE-81675 München (Germany)

Tel.+49894140 6474, E-Mail Christina.Guenter@lrz.tum.de 
medicinal products' and for 'tissue/cell transplantation', production processes often need good manufacturing/ laboratory practice facilities and major financial support, especially in the conduct of clinical trials. For the near future, the processes which will be able to pass through this 'bottleneck' of legislative and economic burden will be mainly protein- or matrix-based therapies and intraoperative cell processing, including autologous (stem) cell transplantation.

\section{Skin Wound Therapies}

As one cannot talk about the skin wound, there does not exist the wound therapy. Fortunately, the majority of all skin wounds heal spontaneously without or with only minimal medical aid. This is due to the fact that the human body, like all other organisms, adapted its regenerative capacity during evolution. The lack of wound healing capacity is not compatible with life. Therefore, we find more or less complex regenerative capacities in all organisms. As a result, special care is only needed if the wound gets more complex, or if it affects deeper structures or the organism itself is in a suppressed condition.

\section{Loss of Epidermis (Superficial)}

An isolated loss of the epidermis, leaving the dermis intact, heals completely and without scar formation within 4-8 days. A typical example would be sunburn. In a situation like this basically no specialized therapy is needed. Nevertheless, a variety of therapeutic options exist. Most available products just have a cooling affect, some also have a local analgesic affect and others just help to keep the wound moist.

\section{Loss of Superficial Dermis (Superficial Dermal)}

Superficial dermal thermal injuries display blister formation. If the blister ground is exposed to air it is extremely painful. Today's state-of-the-art treatment option is the occlusive method. It includes the removal of the blisters and occlusive local therapy.

There exist several treatment options and products (silver nitrate, marfenide, vinegar, iodine, silver sulfadiazine, etc.) which enable microbiological control, but some of these lack enough moisturizing capacity for the wound surface. A widely used product is Flammacine ${ }^{\circledR}$ (silver sulfadiazine), which is simple to handle and has a favorable cost-effectiveness ratio. The disadvantage of this is the (painful) daily dressing change and the drying out of the wound area.

New Strategies in Clinical Care of Skin Wound Healing
In the occlusive method, closure of the wound surface is realized with synthetic membranes under strictly sterile conditions (e.g. Biobrane ${ }^{\circledR}$ or Suprathel). The advantage of these products is that they stay in place until complete wound healing, thus painful dressing changes are no longer needed; nevertheless, frequent wound controls are necessary.

\section{Loss of Deep Dermis (Deep Dermal)}

After a deep dermal injury (a deep second-degree burn), the necrotic superficial layers of the skin have to be removed either biologically or surgically until vital layers are exposed.

After bleeding control, keratinocytes (as solution or as sheets) can be transplanted if enough dermal tissue is preserved and the regenerative capacity of the patient is postulated to be sufficient. Split-skin grafts are used if deeper layers of the dermis are involved. If, after extensive thermal trauma, the remaining nondamaged body surface does not allow for sufficient amounts of split-skin grafts to be taken, temporary skin substitutes such as heterologous or xeno split-skin grafts, or amnion, can be used for a short period of time to prevent both infection and hypertrophic granulation and later scar tissue formation.

\section{Loss of Full Skin (Deep)}

If an acute full-thickness skin defect has occurred (e.g. a third-degree burn), the wound has to be cleaned carefully and all remnants of necrotic skin or foreign bodies have to be removed. In a condition like this the underlying tissue is subjected to infection and trauma since the protecting barriers, dermis and epidermis are lost. Therefore, wound closure is the most important aim. This can be achieved with split-skin graft transplantation or, after pretreatment with a dermis substitute (e.g. Integra ${ }^{\circledR}$ ) and neodermis formation, keratinocytes may be transplanted. If a chronic full-thickness skin defect exists we need a different therapeutic strategy. In chronic wounds an 'antihealing environment' exists and a still not fully understood combination of inhibitory factors prevents healing. In addition, chronic wounds are usually colonized with a multitude of microorganisms and are sometimes even infected. These microorganisms have to be, at least grossly, removed before a wound closure attempt can be made. After cleaning the wound and removing necrotic tissue remnants the wound environment has to be changed from antiproliferate to proproliferate.

Therefore, antiproliferative factors such as metalloproteinases and TNF- $\alpha$ have to be antagonized and the 


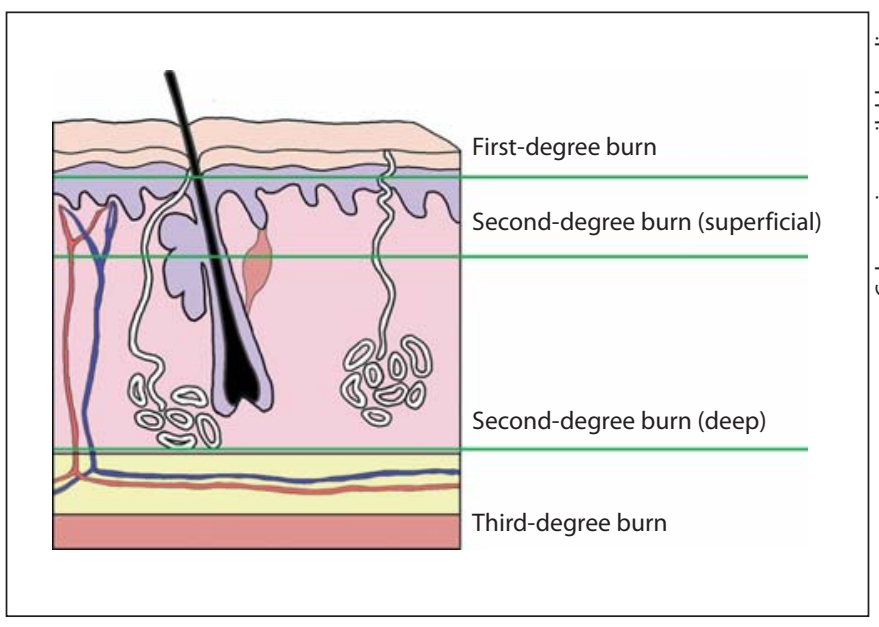

Fig. 1. Four stages of thermal injury to the skin. First-degree burn (spontaneous healing in 5-10 days, no scar formation); Seconddegree burn, superficial (spontaneous healing in 10-21 days, no scar formation); Second-degree burn, deep (spontaneous healing in >21 days, scar formation); Third-degree burn (no spontaneous healing, scar formation).

concentrations and effectiveness of proproliferative factors such as erythropoietin (EPO) or transforming growth factor TGF- $\beta 3$ have to be increased. Granulation tissue formation can then take over or a neodermis can be grown using a dermis substitute. Later split-skin grafts can be transplanted on the prepared new wound bed if necessary. If a proproliferative environment cannot be created, for example due to advanced loss of vital and vascularized tissue, plastic surgical techniques have to be employed by using local or free tissue transfers to substitute the previous tissue loss in an adequate manner.

Figure 1 shows the four stages of thermal injury to the skin.

\section{Innovative Approaches and Clinical Trials}

This section will focus on innovative treatment approaches which are at the stage of clinical phase I-III trials or even only in the preclinical phase, but which seem to have a special promising potential.

\section{Scar-free Healing}

One very interesting fact is the scar-free healing of mammalian embryos. So far, several investigations have been carried out to investigate adult and embryonic wound healing and scarring reaction in adults. Nowa- days, many factors involved in adult and embryonic skin regeneration are being described. In the embryo, the immune system and the inflammatory cascade are not sufficiently developed. Therefore, the resulting inflammatory reaction in the embryo is much smaller and of a shorter period of time than in more advanced developmental stages and adults. Transforming growth factors TGF- $\beta 1-3$ and platelet-derived growth factor (PDGF) seem to play prominent roles. Embryonic scar-free healing can be achieved if PDGF and TGF- $\beta 1$ and 2 are neutralized, and TGF- $\beta 3$ is added to adult wounds [1]. This has already been successfully demonstrated in rodents, pigs and healthy human volunteers [2].

Thus, new drugs for the prevention of scarring are being developed and phases I/II clinical trials have been carried out [3]. Locally administered TGF- $\beta 3$ is well tolerated and improves skin regeneration and thus reduces scarring after trauma [4]. Unfortunately, a multinational, multicenter, double-blind clinical phase III trial testing two different dosing regimens against a placebo was interrupted after 350 patients had been enrolled, and neither the primary nor the secondary study end-points could be met [5].

\section{Regenerative Approaches}

Very few clinical trials with satisfying high evidence levels are to be found in this area of research. This is actually surprising in view of the fact that chronic wounds are the cause of suffering for millions of patients worldwide and cause billions of dollars of costs to the health care systems [6]. One reason might be the difficulty in obtaining standardized and comparable wound conditions in patients, which are needed for proper scientific work.

The only routinely standardized wound in clinical practice is the surgically induced split-skin graft donor site. Therefore, this wound type has already been used as a study target in a multitude of studies to compare different strategies of locally applied therapeutics. None of these, however, has focused on the biological regenerative effects on a cellular level.

If it were possible to activate and deactivate all the tools necessary for wound healing and regeneration, exactly as needed in the particular situation, we would have a universal tool for the acceleration of normal regeneration and wound healing in our hands. However, it has to be taken into consideration that many, especially chronic, wounds are biologically seen far from a normal woundhealing situation. In these instances, therefore, pathological healing processes have to be reduced in favor of biological normalization of the wound milieu. 
Table 1. EPO effects on different growth factors and their most important functions

\begin{tabular}{|c|c|c|c|}
\hline Factor & Effect & Effected cells & EPO action \\
\hline TNF- $\alpha$ & primary injury response & ubiquitary & antagonism \\
\hline IL-2, $-6,-8$ & proinflammatory cytokines & ubiquitary & inhibition \\
\hline$\gamma$-Interferon & proinflammatory cytokine & ubiquitary & inhibition \\
\hline Macrophage inflammatory proteins 1 and 2 & proinflammatory protein & ubiquitary & inhibition \\
\hline Myeloperoxidase & invasion marker for macrophages & ubiquitary & inhibition \\
\hline$\overline{\text { VEGF }}$ & angiogenesis & vessels & stimulation \\
\hline eNOS & recruitment of EPCs & endothelium & stimulation \\
\hline Brain-derived neuroptotic factor & neuronal plasticity & neurones in the CNS & stimulation \\
\hline Thrombopoietin & differentiation & $\begin{array}{l}\text { megakariocytes into } \\
\text { thrombocytes }\end{array}$ & synergism \\
\hline EGF & angiogenesis & endothelium & stimulation \\
\hline BCL-Xl & antiapoptotic & CNS & stimulation \\
\hline Insulin-like growth factor I & antiapoptotic cell proliferation & ubiquitary & stimulation \\
\hline Caspase-3 & apoptosis & ubiquitary & inhibition \\
\hline B cell lymphoma 2 & antiapoptotic proliferation & ubiquitary & stimulation \\
\hline Heat shock protein 70 & intracellular processes & ubiquitary & stimulation \\
\hline
\end{tabular}

\section{Proregenerative Agents}

There are several publications investigating the effects of proregenerative agents on skin regeneration, but few report about their use in humans.

One proregenerative agent which gained increasing attention within the last number of years is EPO. Several proregenerative effects, like anti-inflammatory and antiapoptotic effects, stem cell activation and angiogenesis, could be demonstrated for systemic EPO application in acute and chronic, ischemic and diabetic environments [7-9], as well as for local application in diabetic environments [10]. In a full-thickness-defect mouse model treated with EPO, the healing process clearly improved in a dose-dependent manner [11].

In a standardized murine scalding injury model, the authors could demonstrate statistically significant faster wound healing and reepithelialization after topical EPO application. In addition, the extracellular matrix proliferation was much faster and an increased angiogenesis could be shown with increased CD31, VEGF and eNOS levels. [12].

In the same murine scalding injury model, the combined existence of the EPO receptor and the EPO- $\beta 1$ heteroreceptor in the injured and the noninjured mouse skin could be demonstrated. In the noninjured skin, the receptors were downregulated after EPO treatment, but in the injured skin the receptor expression was stable under EPO treatment. In addition, a faster skin regeneration which was of higher quality could be shown [13].

Even sclerodermic ulcers improved statistically significantly in patients under EPO therapy [14]. Keast and Fraser [15] reported about 4 paraplegic patients whose decubital ulcers improved significantly under systemic EPO treatment.

At present, the first large, prospective, randomized, double-blind, multicenter trial, founded by the German Federal Ministry of Education and Research, is being carried out to investigate the wound-healing effects of EPO in severely burned patients (EudraCT No. 2006-00288638, protocol No. 0506 and ISRCT No. ISRCTN95777824).

Table 1 shows EPO effects on different growth factors and their most important functions.

Another promising approach is the treatment with platelet-rich plasma (PRP) [16-19]. PRP is a biomimetic, highly potential mixture of platelets and multiple growth factors with chemotactic and promitotic qualities [2022]. PRP suppresses proinflammatory cytokines and their actions; it interacts with macrophages, acts proan- 
giogenically and triggers an improved reepithelialization of chronic wounds $[23,24]$. So far, PRP is not part of clinical routine treatment. One reason for this is probably that a certain amount of technical prerequisites are necessary to prepare and use PRP [25]. In addition, the evidence contains lots of contradictory study results and, therefore, it needs further investigation.

\section{Growth Factors}

The use of single or combination growth factors has been investigated concerning their potential for the treatment of chronic wounds. Promising reports in humans were found with epithelial growth factor for the treatment of ulcera cruris [26], and with keratinocyte growth factor [27], fibroblast growth factor [28] and PDGF for the treatment of decubital ulcers [29].

So far, only PDGF has been examined in clinical trials, thus it was used in the treatment of diabetic, neuropathic ulcers. In these trials a significant improvement of wound healing could be demonstrated [30-32]. So far, treatments with growth factors have not reached the clinical routine. The reasons for this are probably of diverse origin, including cost considerations and insufficient scientific evidence; further investigation is, therefore, necessary.

\section{Gene Therapy}

Gene therapy is a possible alternative to the direct application of growth factors. This is because of a continuous or a temporary production and, thus, the effects of the necessary factors can be achieved. In former times, when the biological impact of keratinocytes for creating a stable wound closure was still overestimated, it was demonstrated that transfected keratinocytes are able to survive in a wound and synthesize the respected proteins [33]. Transfected keratinocytes transplanted onto athymic nude mice evoke the desired positive proregenerative effects, but no tendency for malignant degeneration was observed [34]. Nowadays, we know that the prime target for stable wound healing is a sufficiently perfused and stable integrated dermis. Therefore, more scientific attention has recently been directed towards the biological improvement of dermis regeneration and dermal scaffolds (see Tissue Engineering).

There are very few clinical trials being published in the field of gene therapy. In a recently published article the amputation rate of diabetic feet was statistically significantly reduced by the injection of modified endothelial cells into the effected extremity [35].

One of the reasons for the poor evidence situation might be the fact that many gene vectors, especially the viral ones, cause an inflammatory reaction which makes their use in humans highly questionable.

\section{Stem Cells}

The Regulation on Advanced Therapies [regulation (EC) 1394/2007] defines stem cells as advanced therapy medicinal products. Therefore, newly developed production processes and quality procedures have to comply with pharmaceutical standards and good manufacturing practice regulations defined by the European Union, USFDA and ICH. This represents new challenges, and both scientists and the cell-based therapy industry will have to deal with these obstacles in the near future.

Today, dermal stem cells have been identified in the skin, and in skin appendages like hair follicles and sweat glands, which showed the same phenotype as adult mesenchymal stem cells [36-38]. Mesenchymal stem cells, when grown under hypoxic conditions and with the addition of IL- 6 to the culture medium, showed decreased proliferation rates, but when EPO was also added this changed to increased proliferation rates [39].

The first clinical trials were carried out using autologous mesenchymal stem cells (bone marrow). Chronic ischemic wounds treated with bone marrow-derived stem cells revealed complete wound closure in more than $50 \%$ of the patients. Pain reduction could also be achieved as well as a prolongation of walking distance from 0 to 40 $\mathrm{m}[40]$.

CD34+ cells could be successfully applied on diabetic ulcers. After the treatment, the patients revealed improved blood circulation and higher values in transcutaneous oxygen partial pressure [41].

So far, stem cells are not routinely used in the clinical setting, which is at least partially explainable by the above-mentioned regulatory restrictions.

\section{Tissue Engineering}

As mentioned before, skin was the first tissue to be successfully tissue-engineered and implemented back into the clinical routine. The first commercially available tissue-engineered products to be found on the market were keratinocyte sheets.

As already stated, keratinocyte sheets are less commonly used nowadays and serve only as an additive in the therapy of severe full-thickness skin defects. During the first enthusiastic phase, we learned that for the application of keratinocytes an existing dermis (rest) is an obligatory prerequisite, otherwise they cannot expel their bio- 
Table 2. Overview of the literature, preclinical and clinical trials

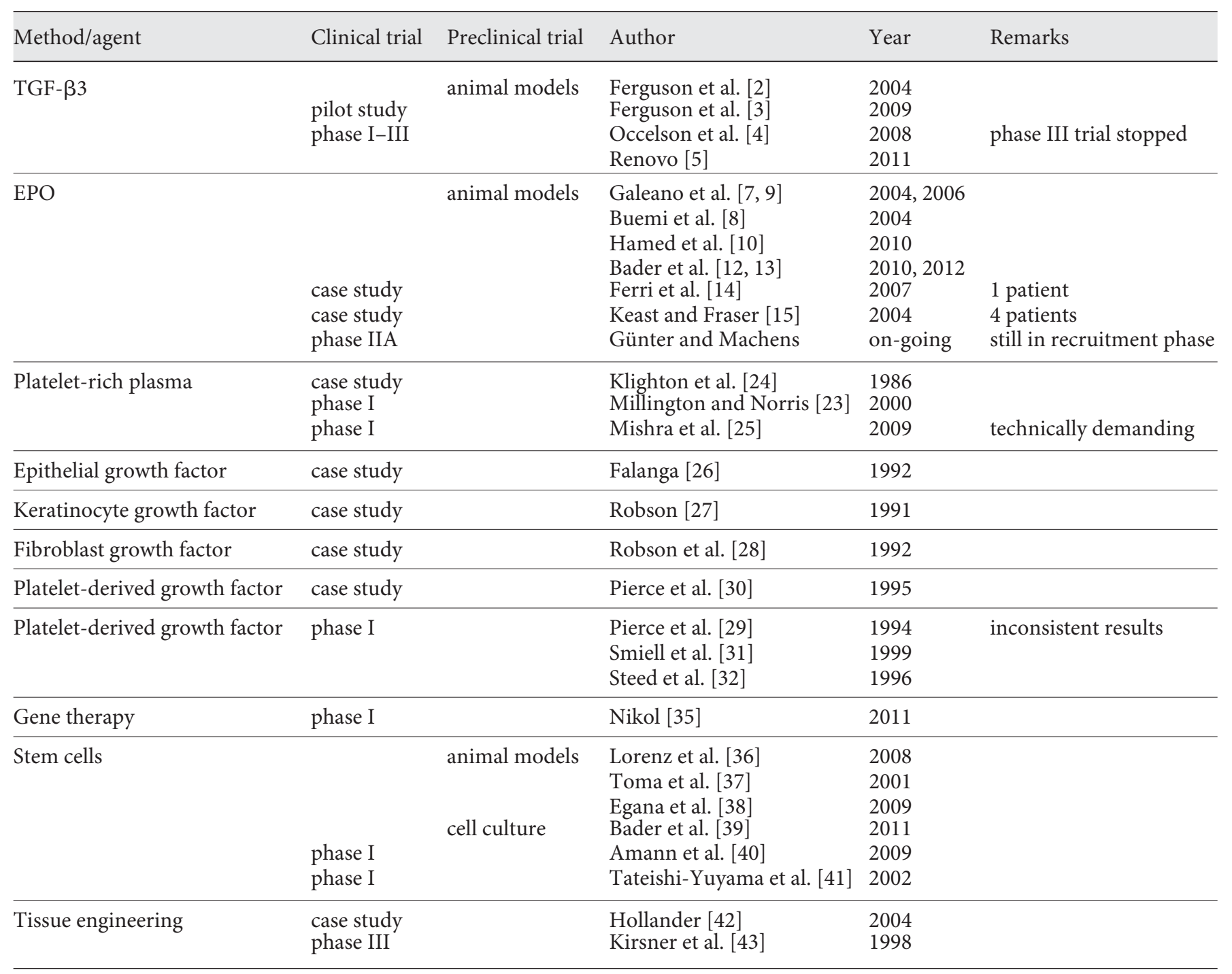

logical effects, which causes impaired healing and scarring. Actually, the most commonly used variety is keratinocyte fibrin spray, which is used especially in dermis-preserved pediatric superficial thermal injuries. Heterologous keratinocytes have additional disadvantages in that they are not only expensive, but they might also transmit infective diseases.

Ideally, a tissue-engineered skin substitute is a multilayered, structurally fully functional skin substitute, which might fulfill all the different functions of the dermis and epidermis.

An interesting multilayer tissue-engineering approach to cover large full-thickness defects has already been de- scribed a few years ago. Autologous keratinocyte and fibroblast primer cell cultures were established, the cells were grown on special hyaluronic acid matrices, and the construct was transplanted in a single-step procedure. A completely autologous and biological fully active epidermal-dermal substitute was realized, and in addition a thin split-skin graft could be transplanted if necessary [42].

Apligraft ${ }^{\circledR}$ is a tissue-engineered multilayered skin equivalent which is commercially available and approved by the legal authorities (FDA and EMEA). It is a bilayered skin substitute consisting of allogenic neonatal fibroblasts and keratinocytes grown in and on a bovine col- 
lagen matrix. Phase III clinical trials testing the product in different chronic wounds (venous ulcers and diabetic foot ulcers) showed very promising results with an $80 \%$ take rate and statistically significant higher healing rates of the chronic wounds. Nevertheless, disadvantages are the allogenic origin of the cells and, therefore, the unclear infectiological status, the bovine origin of the collagen, the lack of dermal structures like sweat glands and hair follicles and the unclear fate of the graft after transplantation in general [43].

See table 2 for an overview of the literature, including preclinical and clinical trials.

\section{Conclusions and Future Perspectives on Skin Regenerative Therapies}

Regenerative therapies after skin injuries, especially with local topical approaches, have been studied for a long time, but in the majority of cases without focusing on the underling biological processes taking place. Only recently, with a better molecular biological understanding of stem cell and protein-based principles, are we able to customize regenerative therapeutic strategies which respect such fundamental biological principles. Perhaps the therapeutic use of proregenerative agents like EPO, which selectively triggers cell-protective and proregenerative effects, or stem cells or combinations of these methods, may play a key role in future developments of new therapeutics to enable and improve regeneration after skin injuries. Full skin loss will still remain a therapeutic challenge for clinicians, since total skin loss necessitates skin transplantation or bioartificial generation of skin substitutes in such situations. Three key problems need to be solved in the future to optimize skin tissue engineering and tissue regeneration: (1) creating a stable epidermo-dermal junction between the two major compartments (dermis and epidermis); (2) implementing a vascular supply in the dermal layer and (3) supporting the construct with its functional cells and appendices (e.g. melanocytes, sweat and sebaceous glands, hair bulges, etc.).

Today, evidence-based treatments for these patients are hardly possible because of insufficient scientific evidence due to the lack of a sufficient number of high-quality clinical trials. To offer evidence-based, cost-effective and up-to-date therapies we need more high-quality clinical trials following the rules of good medical practice and high ethical, moral and scientific standards to be able to identify the most promising new therapeutic methods.

\section{References}

1 Shah M, et al: Neutralisation of TGF- $\beta 1$ and TGF- $\beta 2$ or exogenious addition of TGF- $\beta 3$ to cutaneous rat wounds reduces scarring. J Cell Sci 1995;108:985-1002.

2 Ferguson MWJ, et al: Scar-free healing: from embryonic mechanisms to adult therapeutic intervention. Philos Trans R Soc Lond B 2004;359:839-850.

-3 Ferguson MWJ, et al: Prophylactic administration of avotermin for improvement of skin scarring: three double-blind, placebo-controlled, phase I/II studies. Lancet 2009;373: 1264-1274.

4 Occleston NL, et al: Prevention and reduction of scarring in the skin by Transforming Growth Factor beta 3 (TGFbeta3): from laboratory discovery to clinical pharmaceutical. J Biomater Sci Polym Ed 2008; 19:10471063.

5 Renovo: Juvista EU phase 3 trial results. 2011. www.renovo.com/en/news/juvista-euphase-3-trial-results.

6 Horch RE, et al: Ökonomische Aspekte in der chirurgischen Wundbehandlung. Chirurg 2008;79:518-525.

7 Galeano M, et al: Recombinant human EPO stimulates angiogenesis and wound healing in the genetically diabetic mouse. Diabetes 2004;53:2509-2517.

8 Buemi M, et al: Recombinant human EPO stimulates angiogenesis and healing of ischemic skin wounds. Shock 2004;22:169-173.

$\checkmark 9$ Galeano M, et al: Recombinant human EPO improves angiogenesis and wound healing in experimental burn wounds. Crit Care Med 2006;34:1139-1146.

10 Hamed S, et al: Topical EPO promotes wound repair in diabetic rats. J Invest Dermatol 2010;130:287.

11 Sorg H, et al: Effects of EPO in skin wound healing are dose related. FASEB 2009;23: $1-10$.

12 Bader A, Machens HG: Recombinant human EPO plays a pivotal role as a topical stem cell activator to reverse effects of damage to the skin in aging and trauma. Rejuvenation Res 2010;13:499.

13 Bader A, et al: Skin regeneration with conical and hair follicle structure of deep seconddegree scalding injuries via combined expression of the EPO receptor and beta common receptor by local subcutaneous injection of nanosized rhEPO. Int J Nanomedicine 2012;7:1227-1237.
14 Ferri C, et al: Treatment of severe scleroderma skin ulcers with recombinant human EPO. Clin Exp Dermatol 2007;32:287-290.

15 Keast DH, Fraser C: Treatment of chronic skin ulcers in individuals with anemia of chronic disease using rhEPO: a review of four cases. Ostotomy Wound Manage 2004; 50:64-70.

16 Driver VR, Hanft J, Fylling CP, Beriou JM, Autologel Diabetic Foot Ulcer Study Group: A prospective, randomized, controlled trial of autologous platelet-rich plasma gel for the treatment of diabetic foot ulcers. Ostomy Wound Manage 2006;52:68-70, 72, 74.

17 Pietrzak WS, et al: Platelet rich plasma: biology and new technology. J Cranoiofac Surg 2005;16:1043-1054.

18 Eppely BL, et al: Platelet rich plasma: a review of biology and applications in plastic surgery. Plast Reconstr Surg 2006;118:147e-159e.

19 Lacci KM, et al: Platelet-rich plasma: support for its use in wound healing. Yale J Biol Med 2010;83:1-9.

20 Metha S, Watson TJ: Platelet-rich concentrate: basis science and current clinical applications. J Orthop Trauma 2008;22:432438 . 
21 Marx RE: Platelet-rich plasma (PRP): what is PRP and what is not PRP. Implant Dent 2001; 10:225-228.

22 Eppely BL, et al: Platelet quantifications and growth factor analysis from platelet-rich plasma: implications for wound healing. Plast Reconstr Surg 2004;114:1502-1508.

23 Millington JT, Norris TW: Effective treatment strategies for diabetic foot wounds. J Fam Pract 2000;49:40-48.

24 Klighton DR, et al: Classification and treatment of chronic nonhealing wounds. Successful treatment with autologous plateletderived wound healing factors. Ann Surg 1986;2004:322-330.

25 Mishra A, et al: Treatment of tendon and muscle using PRP. Clin Sports Med 2009;28: 113-125.

26 Falanga V: Classifications for wound bed preparation and stimulation of chronic wounds. Wound Repair Regen 2000;8:347352.

27 Robson MC: Growth factors as wound healing agents. Curr Opin Biotechnol 1991;2: 863-867.

28 Robson MC, et al: The safety and effect of topically applied recombinant basic fibroblast growth factor on the healing of chronic pressure sores. Ann Surg 1992;216:401-406, discussion 406-408.

29 Pierce GF, et al: Tissue repair processes in healing chronic pressure ulcers treated with recombinant platelet-derived growth factor BB. Am J Pathol 1994;145:1399-1410.
30 Pierce GF, et al: Detection of platelet-derived growth factor (PDGF)-AA in actively healing human wounds treated with recombinant PDGF-BB and absence of PDGF in chronic nonhealing wounds. J Clin Invest 1995;96:1336-1350.

31 Smiell JM, et al: Efficacy and safety of becaplermin (recombinant human platelet-derived growth factor-BB) in patients with nonhealing, lower extremity diabetic ulcers: a combined analysis of four randomized studies. Wound Repair Regen 1999;7:335346.

32 Steed DL, et al: Effect of extensive debridement and treatment on the healing of diabetic foot ulcers. Diabetic Ulcer Study Group. J Am Coll Surg 1996;183:61-64.

33 Vogt PM, et al: Genetically modified keratinocytes transplanted to wounds reconstitute the epidemis. Proc Natl Acad Sci USA 1994; 91:9307-9311.

34 Eming SA, et al: Genetically modified human keratinocytes overexpressing PDGF-A enhance the performance of a composite skin graft. Hum Gene Ther 1998;9:529-539.

35 Nikol S: Therapeutische Angiogenese mittels Gen- und Stammzellentherapie bei der peripheren arteriellen Verschlusskrankheit. Dtsch Med Wochenschr 2011;136:672-674.
6 Lorenz K, et al: Multilineage differentiation potential of human dermal skin-derived fibroblasts. Exp Dermatol 2008;17:925-932.

- 37 Toma JG, et al: Isolation of multipotent adult stem cells from the dermis of mammalian skin. Nat Cell Biol 2001;3:778-784.

38 Egana JT, et al: The use of glandular-derived stem cells to improve vascularization in scaffold-mediated dermal regeneration. Biomaterials 2009;30:5918-5926.

39 Bader A, et al: Interactive role of trauma cytokines and EPO and their therapeutic potential for acute and chronic wounds. Rejuvenation Res 2011;14:57-66.

40 Amann B, et al: Extremitätenerhalt durch autologe Knochenmarksstammzelltransplantation zur Induktion der Arteriogenese bei kritischer, nicht-revaskularisierbarer Extremitätenischämie. Zentralbl Chir 2009; 134:298-304.

41 Tateishi-Yuyama E, et al: Therapeutic angiogenesis for patients with limb ischaemia $b$ autologous transplantation of bone-marrow cells: a pilot study and a randomised controlled trial. Lancet 2002;360:427-435.

42 Hollander DA: Reconstruction of extensive soft tissue loss by transplantation of dermal and epidermal equivalents. Zentralbl Chir 2004;129:29-36.

43 Kirsner RS, Falanga V, Eaglstein WH: The development of bioengineered skin. Trends Biotechnol 1998;16:246-249. 